\title{
Celiac Disease in a Patient with Baker's Asthma and Wheat Allergy Due to Tri a 14
}

\author{
Elide Anna Pastorello1*, Maria Gloria Aversano, Ambra Mascheri', Laura Farioli2, \\ Laura Michelina Losappio1, Corrado Mirone1, Donatella Preziosi' ${ }^{1}$, Joseph Scibilia1 \\ ${ }^{1}$ Department of Allergology and Immunology, Niguarda Ca' Granda Hospital, Milan, Italy \\ ${ }^{2}$ Department of Laboratory Medicine, Niguarda Ca' Granda Hospital, Milan, Italy \\ Email: elide.pastorello@ospedaleniguarda.it
}

Received 24 April 2015; accepted 3 July 2015; published 6 July 2015

Copyright (C) 2015 by authors and Scientific Research Publishing Inc.

This work is licensed under the Creative Commons Attribution International License (CC BY). http://creativecommons.org/licenses/by/4.0/

(c) (i) Dpen Access

\section{Abstract}

We describe the case of a patient that developed persistent severe asthma after having started to work in a bakery. The subsequent appearance of gastrointestinal symptoms was diagnosed as celiac disease (CD). She also experienced severe asthma attacks when cooking pasta, and experienced anaphylactic shock a few minutes after wheat flour inhalation. The allergologic workup was positive for several cereal products and Tri a 14 (Tricum aestivum 14), while specific IgE titer to Pru p 3 (Prunus persica 3) was negative. Our patient had no recurrence of these episodes when she avoided cooking wheat flour products and wheat in processed foods. The pathogenic mechanisms underlying CD and IgE-mediated food allergy are different and the coexistence of both diseases seems to be rare. Tri a 14 is the major wheat allergen involved in our case; this allergen can sensitize through the respiratory and the oral route, and can give way to anaphylaxis. A possible role played by interleukin-15 (IL-15) and interleukin-21 (IL-21) in the induction of IgE-mediate hypersensitivity, as well as in the pathogenesis of $C D$, is prospected and discussed briefly herein.

\section{Keywords}

Celiac Disease, Baker's Asthma, Wheat Allergy, Tri a 14, IL-15

\section{Introduction}

Wheat contributes $50 \%$ of the caloric intake in industrialized and developing countries. The major wheat-related disorders include wheat allergy (WA) and gluten related hypersensitivity. Among the allergic reactions to wheat we can identify baker's asthma and food allergy, including wheat dependent exercise induced anaphylaxis

\footnotetext{
${ }^{*}$ Corresponding author.
}

How to cite this paper: Pastorello, E.A., Aversano, M.G., Mascheri, A., Farioli, L., Losappio, L.M., Mirone, C., Preziosi, D. and Scibilia, J. (2015) Celiac Disease in a Patient with Baker's Asthma and Wheat Allergy Due to Tri a 14. Case Reports in Clinical Medicine, 4, 253-256. http://dx.doi.org/10.4236/crcm.2015.47050 
(WDEIA). Members of the $\alpha$-amylase/trypsin inhibitor family and LTP (lipid transfer protein) Tri a 14 behave as major allergens in both food allergy and baker's asthma, while thioredoxins, peroxidase and others represent minor wheat flour allergens [1] [2]. $\omega-5$ gliadin represents the most important allergen in WDEIA; recently wheat LTP also has been identified as causative allergen in WDEIA [3]. In contrast, CD is an autoimmune disorder, characterized by anti-tissue transglutaminase (anti-tTG) autoantibodies. Other wheat induced disorders are the still being discussed non-celiac gluten sensitivity and irritable bowel syndrome worsened by FODMAPs (fermentable oligo-, di-, and mono-saccharides and polyols) [4]. The objective of the paper was to present the case of a patient who developed wheat hypersensitivity by different and contrasting immunological mechanisms.

\section{Case Report}

A 44-year-old woman was referred to our Department in order to investigate the causes of several anaphylactic reactions that occurred a few months before her visit. This patient suffered from grass pollen rhinitis and cat induced asthma; she had developed persistent severe asthma after beginning to work in a bakery, whereas she continued to tolerate ingested wheat products. Subsequently, she stopped her activity as a baker due to the increasing severity of the asthma attacks. The patient subsequently developed gastrointestinal symptoms that directed toward a diagnosis of CD, which was later confirmed by elevated anti-tTG and anti-endomysial antibodies (Table 1) and a typical celiac histology at biopsy of the small intestine. In fact, histology showed villous atrophy, lengthening of the crypts, and an extended increase in the number of intraepithelial lymphocytes (Marsh IIIa). She also began to experience severe asthma attacks when boiling pasta for her family, and manifested anaphylactic shock a few minutes after the inhalation of wheat flour. Another noteworthy episode occurred when she opened a box of pizza and developed facial angioedema, urticaria and acute asthma, requiring the use of selfinjectable epinephrine. The allergologic workup was positive for skin prick tests and specific IgE antibodies for several cereal products and Tri a 14, whereas specific IgE titer to Pru p 3 was negative. Table 1 shows clinical and diagnostic characteristics of our case.

The patient was instructed to avoid cooking of wheat-flour products and wheat in processed foods. In consideration of the severe clinical symptoms we strongly suggested continuous therapy with an inhaled steroid as well as treatment with anti-IgE monoclonal antibodies and to always keep two adrenaline auto-injectors at the disposal. At one-year follow-up she had no recurrence of the wheat-induced episodes.

Table 1. Clinical and diagnostic characteristics of our patient.

\begin{tabular}{|c|c|c|}
\hline \multirow{2}{*}{ Antibodies ( $\mathrm{U} / \mathrm{mL})$} & Anti-tTG & 200 \\
\hline & Anti-endomysial & 60 \\
\hline \multicolumn{2}{|c|}{ Duonenal biopsy } & Positive for CD Marsh IIIa \\
\hline \multicolumn{2}{|c|}{ SPT positive to: } & Wheat \\
\hline \multicolumn{2}{|c|}{ Total IgE (KU/L) } & 439 \\
\hline \multirow{10}{*}{ Specific IgE values (KU } & Wheat & 61.5 \\
\hline & Gluten & 61.6 \\
\hline & Rye & 52 \\
\hline & Barley & 31.1 \\
\hline & Oats & 9.58 \\
\hline & Corn & 1.98 \\
\hline & Brown rice & 0.68 \\
\hline & $\omega-5$ gliadin & $<0.1$ \\
\hline & Tri a 14 & 15 \\
\hline & Pru p 3 & $<0.1$ \\
\hline
\end{tabular}




\section{Discussion}

To our knowledge, this is the first report of an IgE-mediated and non-IgE-mediated wheat hypersensitivity reaction induced by occupational exposure. The pathogenic mechanisms underlying CD and an IgE-mediated WA are different and the coexistence of both diseases seems to be rare. Several published reports have highlighted cases of reactions induced by inhalation of airborne allergens from some foods, such as fish, shellfish, seeds, soybeans, cereal grains and cow milk [5]. The major wheat allergen involved in our case was Tri a 14, which can sensitize either through the respiratory or the oral routes, therefore inducing anaphylaxis. In our case the Tri a 14 sensitization was through the inhalation route. Tri a 14 is a wheat allergen that belongs to the LTP family which is part of the prolamin superfamily and represents one of the major group of food and respiratory allergens. The most important allergen in this family is Pru p 3 that usually gives rise to "LTP syndrome" inasmuch as it causes sensitization to several LTPs contained in plant foods, hence the term "LTP syndrome". However, wheat LTP, Tri a 14, is less cross-reactive with Pru p 3 than other LTPs, due to different aminoacids in critical position of the sequence. Thus the negativity of IgE to Pru p 3 in our patient was not surprising. Moreover, in the literature there are reports of different epitopes of wheat LTP being recognized by baker's asthma patients with respect to peach LTP allergic patients [6].

The problem however is how can one simultaneously account for a Th1 and Th2 mediated disease? We have no experimental data on this problem but on the basis of the literature we can advance some hypotheses. In particular, we suggest that IL-15 together with IL-21 may be implicated in the induction of IgE-mediated hypersensitivity, as well as in the pathogenesis of $\mathrm{CD}$. In CD, intestinal lesions result from the interplay between $\mathrm{CD} 4^{+}$ lamina propria $\mathrm{T}$ cells that produce interferon c (IFNc) in response to gluten peptides presented by human leukocyte antigen-DQ2 ${ }^{+}$antigen-presenting cells and intraepithelial lymphocytes (IELs) activated by IL-15 and innate natural killer receptors. IL-21 may amplify the local Th1 response by inhibiting regulatory T cells. IL-21 may act in synergy with IL-15, thus promoting the activation of IELs, and their cytotoxicity against enterocytes [7]. However, in the pathogenesis of allergic rhinitis and asthma, IL-15 expression may be necessary to develop atopy and asthma, by promoting $\mathrm{CD}^{+} \mathrm{T}$ cells and enhancing the differentiation into Th2 cells in the presence of IL-4 [8] and IL-21 [9]. Moreover, an important role for IL-21 recently has been demonstrated in the mobilization of skin dendritic cells to draining lymphnodes and the subsequent allergic response to epicutaneous introduced antigen. Even though this role has been demonstrated in the atopic dermatitis model, we speculate that prolonged and intensive exposure to wheat dust may have induced enough sensitization to stimulate the production of this cytokine. As matter of fact the CD in our patient arose after the IgE sensitization to wheat and prolonged occupational wheat exposure given that the patient was the owner of the bakery and could not immediately cease activity.

\section{Conclusion}

Wheat hypersensitivity mediated by Tri a 14 sensitisation can cause baker's asthma and food anaphylaxis induced by wheat vapour inhalation.

\section{Acknowledgements}

No grants were received in relation to this paper.

\section{Competing Interests}

The authors declare that they have no competing interests.

\section{Authors' Contributions}

All authors have been involved in drafting the manuscript and revising it critically, giving final approval of the version to be published.

\section{References}

[1] Pastorello, E.A., Farioli, L., Conti, A., Pravettoni, V., Bonomi, S., Iametti, S., et al. (2007) Wheat IgE-Mediated Food Allergy in European Patients: $\alpha$-Amylase Inhibitors, Lipid Transfer Proteins and Low-Molecular-Weight Glutenins. 
International Archives of Allergy and Immunology, 144, 10-22. http://dx.doi.org/10.1159/000102609

[2] Palacin, A., Quirce, S., Armentia, A., Fernández-Nieto, M., Pacios, L.F., Asensio, T., et al. (2007) Wheat Lipid Transfer Protein Is a Major Allergen Associated with Baker's Asthma. Journal of Allergy and Clinical Immunology, 120, 1132-1138. http://dx.doi.org/10.1016/j.jaci.2007.07.008

[3] Pastorello, E.A., Farioli, L., Stafylaraki, C., Scibilia, J., Mirone, C., Pravettoni, V., et al. (2014) Wheat-Dependent Exercise Induced Anaphylaxis Caused by a Lipid Transfer Protein and Not by $\omega-5$ Gliadin. Annals of Allergy, Asthma Immunology, 112, 386-387. http://dx.doi.org/10.1016/j.anai.2014.01.012

[4] Gibson, P.R. and Shepherd, S.J. (2012) Food Choice as a Key Management Strategy for Functional Gastrointestinal Symptoms. American Journal of Gastroenterology, 107, 657-666. http://dx.doi.org/10.1038/ajg.2012.49

[5] James, J.M. and Crespo, J.F. (2007) Allergic Reactions to Foods by Inhalation. Current Allergy and Asthma Reports, 7, 167-174. http://dx.doi.org/10.1007/s11882-007-0017-z

[6] Tordesillas, L.I., Pacios, L.F., Palacin, A., Quirce, S., Armentia, A., Barber, D., et al. (2009) Molecular Basis of Allergen Cross-Reactivity: Non-Specific Lipid Transfer Proteins from Wheat Flour and Peach Fruit as Models. Molecular Immunology, 47, 534-540. http://dx.doi.org/10.1016/j.molimm.2009.07.028

[7] Meresse, B., Verdier, J. and Cerf-Bensussan, N. (2008) The Cytokine Interleukin 21: A New Player in Celiac Disease? Gut, 57, 579-581. http://dx.doi.org/10.1136/gut.2007.141994

[8] Pinto, L.A., Depner, M., Steudemann, L., Klopp, N., Illig, T., von Mutius, E., et al. (2009) IL-15 Gene Variants Are Not Associated with Asthma and Atopy. Allergy, 64, 643-646. http://dx.doi.org/10.1111/j.1398-9995.2008.01830.x

[9] Gong, F., Su, Q., Pan, Y.H., Huang, X. and Shen, W.H. (2013) The Emerging Role of Interleukin-21 in Allergic Diseases. Biomedical Reports, 1, 837-849. 